\title{
Optimal claim behaviour for vehicle damage insurances
}

\author{
N.P. Dellaert and J.B.G. Frenk \\ Econometric Institute, Erasmus University Rotterdam, Rotterdam, Netherlands
}

\author{
L.P. van Rijsoort \\ Coopers \& Lybrand, Utrecht, Netherlands
}

Received December 1990

Revised March 1993

\begin{abstract}
In this paper we analyse the optimal claim behaviour of a risk sensitive policy holder having a vehicle damage insurance. It is proved that the optimal decision is of the form: to claim for damages only if its amount exceeds a certain limit. Moreover, we also derive the optimal stopping rule to terminate the insurance. Finally, some computational results are presented.
\end{abstract}

Keywords: Bonus-malus systems; Automobile insurance; Markov decision processes.

\section{Introduction}

An important feature of premium rating systems for vehicle insurance is the no-claim or bonus-malus principle. This principle is meant to reward policy holders for not making claims during a year; that is, to grant a bonus to a careful driver.

A bonus principle affects the policy holder's decision whether to claim or not in a particular instance. An example of a bonus-malus scheme operative in the netherlands [cf. Goudse Verzekeringen (1988)] is given in Table 1.

In Dellaert et al. (1990) the optimal claim behaviour of a policy holder having a third-party liability car insurance operative under a given bonus-malus scheme is discussed. In the same paper a detailed overview of the literature on this topic is presented. By similar techniques as used in Dellaert et al. (1990) we will analyse in this paper the optimal bchaviour of a policy holder having a vehicle damagc insurance. Sometimes the vehicle damage insurance and the third-party liability insurance are combined in a so-called all-risk insurance. The vehicle damage insurance only covers damages on the policy holder's car and operates under the same bonus-malus table as a third-party liability insurance [cf. Goudse Verzekeringen (1988)]. However, there are some differences.

First of all, contrary to a third-party liability insurance, the vehicle damage insurance is not made obligatory. This implies that the policy holder has the option to join or terminate the insurance and hence in the analysis of the decision process one has to take account of the policy holder's risk sensitivity. For simplicity it is assumed that all insurance companies apply the same rates and so the policy holder does not have the opportunity to terminate the insurance and take a cheaper vehicle damage insurance with another insurance company. 
Table 1

Percentages of the basic premium by bonus-malus class.

\begin{tabular}{|c|c|c|c|c|c|}
\hline \multirow{2}{*}{$\begin{array}{l}\text { Bonus-malus } \\
\text { class }\end{array}$} & \multirow{2}{*}{$\begin{array}{l}\text { Premium as a per- } \\
\text { centage of the } \\
\text { basic premium }\end{array}$} & \multicolumn{4}{|c|}{ New bonus-malus class after .. claims } \\
\hline & & $\overline{0}$ & 1 & 2 & $\geq 3$ \\
\hline 14 & 30.0 & 14 & 9 & 5 & 1 \\
\hline 13 & 32.5 & 14 & 8 & 4 & 1 \\
\hline 12 & 35.0 & 13 & 8 & 4 & 1 \\
\hline 11 & 37.5 & 12 & 7 & 3 & 1 \\
\hline 10 & 40.0 & 11 & 7 & 2 & 1 \\
\hline 9 & 45.0 & 10 & 6 & 1 & 1 \\
\hline 8 & 50.0 & 9 & 5 & 1 & 1 \\
\hline 7 & 55.0 & 8 & 4 & 1 & 1 \\
\hline 6 & 60.0 & 7 & 3 & 1 & 1 \\
\hline 5 & 70.0 & 6 & 2 & 1 & 1 \\
\hline 4 & 80.0 & 5 & 1 & 1 & 1 \\
\hline 3 & 90.0 & 4 & 1 & 1 & 1 \\
\hline 2 & 100.0 & 3 & 1 & 1 & 1 \\
\hline 1 & 120.0 & 2 & 1 & 1 & 1 \\
\hline
\end{tabular}

Secondly, if a total loss occurs and a claim is submitted, the indemnity paid by the insurance company depends on the age of the car. Hence the older the car gets, the less attractive it will be for the policy holder to be insured, since at the same time the bonus-malus scheme with its basic premium does not change. These two major differences complicate the analysis of the decision process.

In the next section the assumptions and the mathematical model describing the decision process will be introduced and analysed. Moreover, in Section 3 some computational results for an existing vehicle damage insurance will be presented.

\section{The model}

In this section we introduce a discrete Markov decision process to model the vehicle damage insurance. However, before discussing the model, we specify the following assumptions. Some of the used notions will be defined and explained in detail later in this section.

- A policy holder always buys a new car of approximately the same list price and with approximately the same damage distribution if his present car is a 'total loss' or if it reaches a given age $U$.

- A policy holder faces a deduction of size $f$. This means, if he claims for a damage, he has to pay the amount $f$ himself.

Clearly the second assumption is realistic. To discuss the first assumption we first observe that the decision of buying another car and which type of car is not determined within the model. This decision clearly depends on other factors such as the wealth and preference of the policy holder. However, in order to determine the policy holder's optimal behaviour with respect to his vehicle damage insurance we need to know in advance under which replacement rule the policy holder operates. As an example of another replacement rule we mention the rule that a policy holder always buys a second-hand car bclonging to a certain category after a 'total loss' or after the present car reaches a certain age. In principle, all replacement rules which uniquely determine the future bonus-malus class and its corresponding premium, given the present premium, can be incorporated into the model. Also, since the model determines an average optimal decision based on random information about the sizes of future damages and appearances of future accidents we need to know in advance the so-called damage distribution of the (future) policy holder's car. Clearly the repair costs and damage characteristics of cars belonging to a different category might be completely different. Finally, in order to decide whether a car 
is a 'total loss' we must know an estimate of the current value of the car before an accident and its demolition value after the accident. These estimates also depend on the type of car. The first assumption was introduced to keep the model simple with respect to the necessary information. Roughly this assumption implies that we only need to know one function for the current value, one list price and one damage distribution. However, if in some cases this assumption deviates too much from the replacement rule of an arbitrary policy holder we can introduce this more specific replacement rule. In general the model can handle more accurate rules at the cost of computational efficiency and a more complicated notation. Having in mind that a model is always an inaccurate description of reality we might argue that a possible advantage does not offset the complications and so we should use only very simple replacement rules like the one mentioned above. Finally, the authors believe that a more severe restriction of this model is its incompetence to handle different competing insurance companies and to model the 'shopping' behaviour of policy holders to different companies. To model this a somewhat different model is needed and this will be the topic of future research.

Now, in order to derive the Markov decision model we need to define the set of decision moments, the decision space, the state space and the transition probabilities.

Let the decision process continue $T$ years $(T<\infty)$ and start at the beginning of a certain year. We divide an insurance period, generally a year, into $N$ equal subperiods. We assume that there can only be one accident during a subperiod and that the decision to claim or not to claim for a particular damage has to be taken at the end of a subperiod. Then the set of decision moments is defined as

$$
\mathscr{T}:=\{t \mid t=1,2, \ldots, H\} \text { where } H=N T .
$$

A policy holder has to take two kinds of decisions. The first is whether he claims for a damage or not. This decision is modelled by the variable $d_{1}\left(d_{1}=0,1\right)$, where 1 denotes to claim and 0 denotes not to claim.

The second decision deals with the opportunity to continue or to terminate the insurance. Only at the end of each insurance year, a policy holder can decide whether he terminates the insurance at the end of that year or not. Moreover, if the policy holder is not insured at the end of a certain subperiod, he can always decide to join the insurance. Consequently, he only pays a part of the premium, accordant with that part of the year during which he is insured. This second decision is given by the variable $d_{2}$ $\left(d_{2}=0,1,2\right)$, where 0 denotes no transition, 1 denotes terminating the insurance at the end of the year and 2 denotes effecting an insurance at the end of a subperiod. By these observations it is clear that the decision space is given by

$$
\mathscr{D}:=\left\{\left(d_{1}, d_{2}\right) \mid d_{1}=0,1 ; d_{2}=0,1,2\right\} .
$$

At each decision moment, the policy holder faces a state $E_{t}$. This state consists of six components, of which some will be explained in the remainder. These components are:

$j=$ the policy holder's bonus / malus combination $\left(\pi_{0}(j), \pi_{1}(j)\right), j=1, \ldots, J$;

$i=$ the index defining a year, $0 \leq i \leq T-1$;

$n=$ the index defining a subperiod, $1 \leq n \leq N$;

$l=$ the car's age, $1 \leq l \leq U$ with $U$ representing the total time the policy holder keeps his car;

$c=\{0,1\}$, where 0 denotes not being insured, and 1 denotes being insured;

$x=$ the amount of damage resulting from an accident occurring in subperiod $n$. Clearly $x$ equals 0 if no accident occurs;

and so every state $E_{t}$ at time $t$ is given by the following vector:

$$
E_{t}=(j, i, n, l, c, x) \text {. }
$$

We are now going to look at the components $j, c$ and $l$ more closely. The variable $x$ will be treated later on, when stating the transition probabilities. 
The component $j=\left(\pi_{0}(j), \pi_{1}(j)\right)$ consists of

1. the premium $\pi_{0}(j)$ to be paid next year by the policy holder when he does not claim for a damage during the rest of the current year,

2. the premium $\pi_{1}(j)$ to be paid next year by the policy holder when he claims for exactly one damage during the rest of the current year.

As will appear below, the premium that he would pay when he would claim two or more times during the rest of the current year, does not play any role.

We can elicit every possible combination of premiums to be paid from the transition table of the bonus-malus scheme; let there be $J$ such combinations. We denote a particular combination with $j$, where $j=1, \ldots, J$.

We consider two cases where $j$ changes.

1. The policy holder files a claim in one of the subperiods $1, \ldots, N-1$. Observe, if he does not file a claim, the component $j=\left(\pi_{0}(j), \pi_{1}(j)\right)$ will not change. However, if a claim is filed, we denote by $a(j)$ the number of the new combination when his present combination is $j$ and so he moves to the new combination $a(j)=\left(\pi_{0}(a(j)), \pi_{1}(a(j))\right)$ with $\pi_{0}(a(j))=\pi_{1}(j)$.

This transition can be represented schematically by

$$
j \rightarrow\left\{\begin{array}{ll}
j & \text { no claims } \\
a(j) & \text { one claim }
\end{array} .\right.
$$

It follows that it is not necessary to keep record of the number of filed claims, because the next year premium is adapted immediately after a claim is filed.

2. A new insurance year takes effect. The policy holder pays the premium at the beginning of each insurance year. This premium is equal to $\pi_{1}(j)$ or $\pi_{0}(j)$, depending on whether he has or has not filed a claim in state $(j, i, N, l, c, x)$. When he does not file a claim during the new insurance year, he moves to a higher bonus-malus class (unless he is already in the highest class), and for the year thereafter he pays a lower premium than for the starting new year. This corresponds to a different combination $j$ of premiums to be paid.

Consequently, for each $j$ there are two transitions between the subperiods $n=N$ of any year and $n=1$ of the next year:

$$
\left\{\begin{array}{ll}
j \rightarrow j \rightarrow b(j) & \text { if no claim is filed } \\
j \rightarrow a(j) \rightarrow b(a(j)) & \text { if a claim is filed }
\end{array},\right.
$$

where $b(j)$ is defined as the number of the new combination as a result of the transition to the new year. Given a transition mechanism, as presented in Table 1 , we can determine the values of $j, a(j), b(j)$ and of the corresponding premiums. Table 2 contains these values for the 17 different combinations which can be formed from Table 1 , starting with $(1,1)$ corresponding with $j=1$ until $(14,9)$ for $j=17$. For simplicity the components of the vector do not denote the premiums to be paid but the numbers of the corresponding bonus-malus classes. We notice that different values will result when a different bonus-malus system is effective.

The component $c$ of the state space, denoting whether the policy holder is insured or not, can assume the values 0 or 1 . Some changes on $c$ are possible, dependent on the decision moment $t$. Let $t=N i+n$, $0 \leq i \leq T-1$ and $1 \leq n \leq N$. We distinguish the following situations: 
Table 2

Bonus/malus combinations. ${ }^{\text {a }}$

\begin{tabular}{|c|c|c|c|c|}
\hline$j$ & $\rho_{0}(j)$ & $\rho_{1}(j)$ & $a(j)$ & $b(j)$ \\
\hline 1 & 120.0 & 120.0 & 1 & 2 \\
\hline 2 & 100.0 & 120.0 & 1 & 3 \\
\hline 3 & 90.0 & 120.0 & 1 & 4 \\
\hline 4 & 80.0 & 120.0 & 1 & 5 \\
\hline 5 & 70.0 & 120.0 & 1 & 7 \\
\hline 6 & 60.0 & 120.0 & 1 & 9 \\
\hline 7 & 60.0 & 100.0 & 2 & 9 \\
\hline 8 & 55.0 & 100.0 & 2 & 10 \\
\hline 9 & 55.0 & 90.0 & 3 & 10 \\
\hline 10 & 50.0 & 80.0 & 4 & 11 \\
\hline 11 & 45.0 & 70.0 & 5 & 12 \\
\hline 12 & 40.0 & 60.0 & 6 & 13 \\
\hline 13 & 37.5 & 55.0 & 8 & 14 \\
\hline 14 & 35.0 & 55.0 & 9 & 15 \\
\hline 15 & 32.5 & 50.0 & 10 & 16 \\
\hline 16 & 30.0 & 50.0 & 10 & 17 \\
\hline 17 & 30.0 & 45.0 & 11 & 17 \\
\hline
\end{tabular}

a The functions $\rho_{0}(j)$ and $\rho_{1}(j)$ equal $\pi_{0}(j)$ and $\pi_{1}(j)$ as a percentage of the basic premium.

(a) $0 \leq i \leq T-1,1 \leq n \leq N-1$. At the end of any subperiod the policy holder can make the transition of not being insured to being insured. However, he cannot terminate his insurance. Schematically:

$$
\left\{\begin{array}{l}
0 \rightarrow 0,1 \\
1 \rightarrow 1
\end{array}\right.
$$

(b) $0 \leq i \leq T-2, n=N$. At the end of a year, which is not the last year the policy holder is driving a car, the policy holder can decide whether he will or will not be insured during the next year. Schematically:

$$
\left\{\begin{array}{l}
0 \rightarrow 0,1 \\
1 \rightarrow 1,0
\end{array}\right. \text {. }
$$

Before discussing the component $l$ of the state space, we first need to consider the probability distributions of the number of accidents and the amount of damage. We assume that the size of $N$, the number of subperiods in which the total insurance year is divided, is such that the probability of two or more accidents in any subperiod is negligible. Moreover, we assume that the probability of one accident in a certain subperiod may vary from one subperiod to another and that the appearances of accidents in the different subperiods are independent.

We define the probability

$$
p_{n}:=\operatorname{Pr}\{\text { 'one accident in subperiod } n \text { ' }\}, \quad n=1, \ldots, N,
$$

and a random variable $\underline{Z}_{n}$, where

$$
\underline{Z}_{n}:=\left\{\begin{array}{lll}
0 & \text { if } & \text { no accidents occur in subperiod } n \\
1 & \text { if } & \text { one accident occurs in subperiod } n
\end{array} .\right.
$$

Observe that in this paper random variables are denoted by underlined capitals and so by definition we have

$$
\left\{\begin{array}{l}
\operatorname{Pr}\left\{\underline{Z}_{n}=0\right\}=1-p_{n} \\
\operatorname{Pr}\left\{\underline{Z}_{n}=1\right\}=p_{n}
\end{array}\right.
$$


Accidents generally imply damage. We define $\underline{Y}_{n}$ as the amount of damage resulting from an accident in subperiod $n$ and assume the positive random variables $\underline{Y}_{n}$ to be stochastically independently distributed. Since the amount of damage is equal to the cost of repair which is of course bounded from above by the value of a new car, we define

$$
0<\underline{Y}_{n} \leq L,
$$

where $L$ denotes the list price of a new car.

Clearly, if $\underline{X}_{n}$ denotes the total amount of damage in subperiod $n$, we obtain

$$
\underline{X}_{n}=\left\{\begin{array}{lll}
\underline{Y}_{n} & \text { if } & \underline{Z}_{n}=1 \\
0 & \text { if } & \underline{Z}_{n}=0
\end{array},\right.
$$

and hence with $F_{n}(x):=\operatorname{Pr}\left\{\underline{Y}_{n} \leq x\right\}$ satisfying $F_{n}(L)=1$ and $F_{n}(0)=0$ the distribution $G_{n}$ of the random variable $\underline{X}_{n}$ is given by

$$
\begin{aligned}
G_{n}(x) & =\operatorname{Pr}\left\{\underline{X}_{n} \leq x\right\} \\
& =\operatorname{Pr}\left\{\underline{X}_{n} \leq x \mid \underline{Z}_{n}=0\right\} \operatorname{Pr}\left\{\underline{Z}_{n}=0\right\}+\operatorname{Pr}\left\{\underline{X}_{n} \leq x \mid \underline{Z}_{n}=1\right\} \operatorname{Pr}\left\{\underline{Z}_{n}=1\right\} \\
& =1-p_{n}+p_{n} F_{n}(x) .
\end{aligned}
$$

It is now possible to discuss the component $l$. This component, denoting the car's age determines the current value of the car. This current value plays an important role in the decision to continue or to terminate an insurance at the end of the insurance year, and to determine whether a car is a 'total loss' or not. We denote the current value of the car by the decreasing function $h(l), l=1, \ldots, U$ of which an example is presented in Figure 1. The age $l$ is reproduced in subperiods.

To define a 'total loss' we first give an estimate of the demolition value of a car involved in an accident.

Since this demolition value $\operatorname{dem} v(l, x)$ clearly depends on the age $l$ of the car and the amount of damage $x \leq L$ with $L$ the list price of a new car and this value is decreasing in $x$ and $l$ we have chosen the following simple estimate:

$$
\operatorname{dem} v(l, x):=\operatorname{Ch}(l)\left(\frac{L-x}{L}\right)
$$

with $C<1$ some fixed constant.

If an accident occurs with damage $x>0$ and the car involved has age $l$ it is declared a 'total loss' if

$$
x+\operatorname{dem} v(l, x) \geq h(l) .
$$

This implies that the threshold value $\operatorname{tr}(l)$ for a total loss is given by

$$
x \geq \operatorname{tr}(l):=\frac{\operatorname{Lh}(l)(1-C)}{L-C h(l)} .
$$

Although the cost of buying a new car is a direct consequence of a 'total loss' we do not assign these costs to the unexpected loss. Due to the accident the value depreciation of the car is given by

$$
w(l, x):=h(l)-\operatorname{dem} v(l, x)
$$

and so in case of a 'total loss' and no claiming the policy holder's direct costs assigned to the unexpected loss equals $w(l, x)$. Similarly, in case of no 'total loss' and no claiming these costs are given by $x$. Hence, if no claiming occurs, the policy holder's direct costs assigned to the unexpected loss equals $c(l, x)$ with

$$
c(l, x):=\left\{\begin{array}{lll}
x & \text { if } & x<\operatorname{tr}(l) \\
w(l, x) & \text { if } & x \geq \operatorname{tr}(l)
\end{array} .\right.
$$


On the other hand, if claiming occurs, the policy holder's direct costs equal $f$. This concludes our discussion of the direct costs assigned to an accident and the definition of a 'total loss'.

With the probability distribution function for $\underline{X}_{n}$ we can derive the transition probabilities for all possible combinations of states and decisions. That is, we must determine the probability density function for entering state $E_{t+1}$, given that the policy holder was in state $E_{t}$ at the previous point of time $t$ and had taken decisions $d_{1}$ and $d_{2}$. This function is denoted by $h\left(E_{t+1} \mid E_{t}, d_{1}, d_{2}\right)$ and equals $g_{n+1}(x)$ at subperiods 1 to $N-1$ and $g_{1}(x)$ at subperiod $N$. The function $g_{n}(\cdot)$ is the probability density function belonging to the random variable $\underline{X}_{n}$.

Furthermore we define $c\left(E_{t}, d_{1}, d_{2}\right)$ as the direct cost when decisions $d_{1}$ and $d_{2}$ have been taken in the current period and the policy holder is in state $E_{t}$. The values of the direct cost are given in Table 3 in the case of no 'total loss'. Notice, in case of 'total loss' that the corresponding table is identical except that $l^{*}:=l(\bmod \mathrm{U})+1$ is replaced by 1 since in that case the policy holders buys a new car. Also observe, if a policy holder decides to join the vehicle damage insurance and he used to be in combination $j$ that it is assumed that he will continue in $j$ within one insurance year or start in $b(j)$ at the end of an insurance year. However, depending on the policy of the insurance company he might also move to another combination (e.g. bonus is lost after two years without vehicle damage insurance). This policy can also be incorporated by adapting every time the component $j$ according to the company's policy in case of no vehicle damage insurance. Since this requires a specific knowledge of a company's policy we take for simplicity the first option.

Since the policy holder can decide not to join the insurance we have to introduce risk sensitivity into the model. In the Markov decision model we will therefore use an exponential disutility function $V(x)$, describing the risk aversion of a person, as discussed by Howard and Matheson (1972). The disutility function $V(x)=\mathrm{e}^{\alpha x}(\alpha>0)$, measuring the discomfort of a policy holder, is monotone increasing, convex and satisfies the delta-property [cf. Howard and Matheson (1972)]. That means, the policy holder is risk-averse and decides independent of his wealth or income. Howard and Matheson prove that the

Table 3

$c\left(E_{l}, d_{1}, d_{2}\right)$ for all $E_{t}, E_{t+1}, d_{t}, d_{2}$ and no 'total loss'. '

\begin{tabular}{|c|c|c|c|c|}
\hline & $\dot{a}_{1}$ & $d_{2}$ & $E_{t+1}$ & $c\left(E_{t}, d_{1}, d_{2}\right)$ \\
\hline 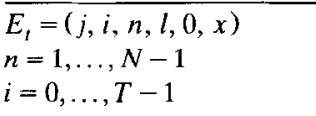 & $\begin{array}{l}0 \\
0\end{array}$ & $\begin{array}{l}0 \\
2\end{array}$ & $\begin{array}{l}\left(j, i, n+1, l^{*}, 0, y\right) \\
\left(j, i, n+1, l^{*}, 1, y\right)\end{array}$ & $\begin{array}{l}c(l, x) \\
c(l, x)+\pi_{0}(j)((N-n) / N)\end{array}$ \\
\hline $\begin{array}{l}E_{i}=(j, i, n, l, 1, x) \\
n=1, \ldots, N-1 \\
i=0, \ldots, T-1\end{array}$ & $\begin{array}{l}1 \\
0\end{array}$ & $\begin{array}{l}0 \\
0\end{array}$ & $\begin{array}{l}\left(a(j), i, n+1, l^{*}, 1, y\right) \\
\left(j, i, n+1, l^{*}, 1, y\right)\end{array}$ & $\begin{array}{l}f \\
c(l, x)\end{array}$ \\
\hline $\begin{array}{l}E_{t}=(j, i, N, l, 0, x) \\
n=N \\
i=0, \ldots, T-2\end{array}$ & $\begin{array}{l}0 \\
0\end{array}$ & $\begin{array}{l}0 \\
2\end{array}$ & $\begin{array}{l}\left(j, i+1,1, l^{*}, 0, y\right) \\
\left(b(j), i+1,1, l^{*}, 1, y\right)\end{array}$ & $\begin{array}{l}c(l, x) \\
c(l, x)+\pi_{0}(j)\end{array}$ \\
\hline $\begin{array}{l}E_{t}=(j, i, N, l, 1, x) \\
n=N \\
i=0, \ldots, T-2\end{array}$ & $\begin{array}{l}1 \\
1 \\
0 \\
0\end{array}$ & $\begin{array}{l}0 \\
1 \\
0 \\
1\end{array}$ & $\begin{array}{l}\left(b(a(j)), i+1,1, l^{*}, 1, y\right) \\
\left(a(j), i+1,1, l^{*}, 0, y\right) \\
\left(b(j), i+1,1, l^{*}, 1, y\right) \\
\left(j, i+1,1, l^{*}, 0, y\right)\end{array}$ & $\begin{array}{l}f+\pi_{0}(a(j)) \\
f \\
c(l, x)+\pi_{0}(j) \\
c(l, x)\end{array}$ \\
\hline $\begin{array}{l}E_{t}=(j, T-1, N, l, 0, x) \\
n=N \\
i=T-1\end{array}$ & 0 & 0 & $\left(j, T, 1, l^{*}, 0, y\right)$ & $c(l, x)$ \\
\hline $\begin{array}{l}E_{t}=(j, T-1, N, l, 1, x) \\
n=N \\
i=T-1\end{array}$ & $\begin{array}{l}1 \\
0\end{array}$ & $\begin{array}{l}1 \\
1\end{array}$ & $\begin{array}{l}\left(a(j), T, 1, l^{*}, 0, y\right) \\
\left(j, T, 1, l^{*}, 0, y\right)\end{array}$ & $\begin{array}{l}f \\
c(l, x)\end{array}$ \\
\hline
\end{tabular}

${ }^{a}$ For the other transitions the transition probabilities equal zero. 
optimal strategy $\sigma$ exists and satisfies

$$
v_{\sigma}\left(E_{0}\right)=\inf _{\gamma} v_{\gamma}\left(E_{0}\right)
$$

where $v_{\gamma}\left(E_{0}\right)$ equals the total expected disutility for the policy holder if his initial state is given by $E_{0}$, and the strategy $\gamma$ is used, i.e.

$$
v_{\gamma}\left(E_{0}\right):=\mathbb{E}\left(\exp \left(\alpha \sum_{t=0}^{N T} \beta^{t} c\left(\underline{E}_{t}, \gamma\right)\right)\right), \quad \alpha>0,0<\beta \leq 1
$$

with $c\left(\underline{E}_{t}, \gamma\right)$ the direct costs in period $t$ if the random state $\underline{E}_{t}$ is observed and $\gamma$ is the used strategy while $\mathbb{E}$ denotes the mathematical expectation symbol. Observe that the index of absolute risk aversion is given by $\alpha$ and that the direct cost occurring during the decision process is discounted by a discount factor $\beta \leq 1$.

In order to illustrate risk aversion, imagine an individual who faces two possible results of a game:

- he wins Dfl.14 with probability 0.5 ,

- he loses Dfl.2 with probability 0.5 .

The expected value $\mathbb{E}[X]$ of this game is Dfl.6. When someone asks him to choose between the certainty equivalent Dfl.6 and the game, the individual is risk-loving if he plays, risk-neutral if he is indifferent, risk-averse if he accepts the Dfl.6. By means of a risk-aversion index $\alpha$ in the disutility function, it is possible to give shape to the degree of risk aversion.

Given the above definitions we are now able to formulate the optimality equations. Introduce therefore for every $1 \leq i \leq T-1,1 \leq n \leq N, 1 \leq l \leq U, c=0,1, x \geq 0$ and $\alpha>0$ :

$$
\begin{aligned}
V_{i}(\alpha, j, n, l, c, x):= & \text { minimal expected total discounted disutility from subperiod } N i+n \\
& \text { through subperiod } N T \text { when the risk-aversion index equals } \alpha \text { and the } \\
& \text { policy holder is in state }(j, i, n, l, c, x) \text { at the end of subperiod } N i+n,
\end{aligned}
$$

and

$$
V_{i}(\alpha, j, n, l, c):=\mathbb{E}\left[V_{i}\left(\alpha, j, n, l, c, \underline{X}_{n}\right)\right] .
$$

In order to derive the optimality equations we consider six possible situations (see Table 4).

Although in each situation a similar reasoning can be applied to derive the optimality equation we distinguish these six different cases to avoid a complicated notation. Observe, since in case of a total loss the policy holder always buys a new car that we need to introduce for the age component $l$ of the state space the function

$$
L(l, x):=\left\{\begin{array}{lll}
l(\bmod U)+1 & \text { if } & x<\operatorname{tr}(l) \\
1 & \text { if } & x \geq \operatorname{tr}(l)
\end{array} .\right.
$$

Case I. If this holds $(0 \leq i \leq T-1,1 \leq n \leq N-1, l \leq U, c=0)$ the policy holder, not being insured, pays in case of an accident the direct $\operatorname{costs} c(l, x)$ himself. He only has to decide whether he will or will nut be insured during the remaining part of the insurance year. This implies that the optimality equation

Table 4

Possible situations.

\begin{tabular}{lll}
- & $\begin{array}{l}c-0 \\
\text { (not insured) }\end{array}$ & $\begin{array}{l}c=1 \\
\text { (insured) }\end{array}$ \\
\hline $0 \leq i \leq T-1,1 \leq n \leq N-1$ & I & II \\
$0 \leq i \leq T-2, n=N$ & III & IV \\
$i=T-1, n=N$ & V & VI \\
\hline
\end{tabular}


has the following form:

$$
V_{i}(\alpha, j, n, l, 0, x)=\exp (\alpha c(l, x)) \min \left\{\begin{array}{l}
V_{i}(\alpha \beta, j, n+1, L(l, x), 0) \\
\exp \left(\alpha \pi_{0}(j) \frac{N-n}{N}\right) V_{i}(\alpha \beta, j, n+1, L(l, x), 1)
\end{array}\right.
$$

Case II. If this holds $(0 \leq i \leq T-1,1 \leq n \leq N-1, l \leq U, c=1)$ the policy holder claims for a damage or not. This implies

$$
V_{i}(\alpha, j, n, l, 1, x)=\min \left\{\begin{array}{l}
\exp (\alpha f) V_{i}(\alpha \beta, a(j), n+1, L(l, x), 1) \\
\exp (\alpha c(l, x)) V_{i}(\alpha \beta, j, n+1, L(l, x), 1)
\end{array} .\right.
$$

Case III. If this holds $(0 \leq i \leq T-2, n=N, l \leq U, c=0)$ the policy holder has to pay in case of an accident the direct costs $c(l, x)$ himself. He only has to decide whether he will or will not be insured during the next insurance year. As such this situation is similar to case I and so we obtain

$$
V_{i}(\alpha, j, N, l, 0, x)=\exp (\alpha c(l, x)) \min \left\{\begin{array}{l}
V_{i+1}(\alpha \beta, j, 1, L(l, x), 0) \\
\exp \left(\alpha \pi_{0}(j)\right) V_{i+1}(\alpha \beta, b(j), 1, L(l, x), 1)
\end{array} .\right.
$$

Case IV. If this holds $(0<i<T-2, n=N, l<U, c=1)$ the policy holder, being insured, decides whether to claim his damage and whether he should continue his insurance. This implies

$$
V_{i}(\alpha, j, N, l, 1, x)=\min \left\{\begin{array}{l}
\exp (\alpha f) \min \left\{\begin{array}{l}
\exp \left(\alpha \pi_{0}(a(j))\right) V_{i+1}(\alpha \beta, b(a(j)), 1, L(l, x), 1) \\
V_{i+1}(\alpha \beta, a(j), 1, L(l, x), 0)
\end{array},\right. \\
\exp (\alpha c(l, x)) \min \left\{\begin{array}{l}
\exp \left(\alpha \pi_{0}(j)\right) V_{i+1}(\alpha \beta, b(j), 1, L(l, x), 1) \\
V_{i+1}(\alpha \beta, j, 1, L(l, x), 0)
\end{array} .\right.
\end{array}\right.
$$

Case V. If this holds ( $i=T-1, n=N, c=0$ ) the policy holder pays in case of an accident his direct costs $c(l, x)$. Since this is the last subperiod he is driving a car he does not have to pay after this subperiod and hence

$$
V_{T-1}(\alpha, j, N, l, 0, x)=\exp (\alpha c(l, x)) .
$$

Case VI. If this holds $(i=T, n=N, c=1)$ the policy holder does not have to pay after this subperiod and so he claims if and only if $c(l, x)>f$. This implies

$$
V_{T-1}(\alpha, j, N, l, 1, x)=\min \left\{\begin{array}{l}
\exp (\alpha f) \\
\exp (\alpha c(l, x))
\end{array} .\right.
$$

The above case concludes the analysis of the claim behaviour of a policy holder. For the cases II, IV and VI, to which the question 'to claim or not to claim' is relevant, we are now able to derive the critical claim sizes. The following expressions can be interpreted as such and this interpretation will be proved in Theorem 1. Introduce for $0 \leq i \leq T-1,1 \leq n \leq N-1, c=1, x>0$ and $l \leq U$,

$$
D_{i}(\alpha, j, n, l, 1, x):=\frac{1}{\alpha} \log \left(\frac{V_{i}(\alpha \beta, a(j), n+1, L(l, x), 1)}{V_{i}(\alpha \beta, j, n+1, L(l, x), 1)}\right) .
$$


Moreover, for $0 \leq i \leq T-2, n=N, l \leq U, c=1, x>0$, let

$$
D_{i}(\alpha, j, N, l, 1, x):=\frac{1}{\alpha} \log \left(\frac{\min \left\{\begin{array}{l}
\exp \left(\alpha \pi_{0}(a(j))\right) V_{i+1}(\alpha \beta, b(a(j)), 1, L(l, x), 1) \\
V_{i+1}(\alpha \beta, a(j), 1, L(l, x), 0)
\end{array}\right)}{\min \left\{\begin{array}{l}
\exp \left(\alpha \pi_{0}(j)\right) V_{i+1}(\alpha \beta, b(j), 1, L(l, x), 1) \\
V_{i+1}(\alpha \beta, j, 1, L(l, x), 0)
\end{array}\right),}\right.
$$

and finally for $i=T-1, n=N, l \leq U, c=1$ and $x>0$ introduce

$$
D_{T-1}(\alpha, j, N, l, 1, x):=0 \text {. }
$$

Although these critical claim sizes depend on $x$ they assume at most two different values, i.e. one for $x \geq \operatorname{tr}(l)$ and one for $x<\operatorname{tr}(l)$.

We are now able to prove the following result.

Theorem 1. Consider an insured policy holder with a risk-aversion index $\alpha$ and a deduction $f$ being in state $(j, i, n, l, 1, x)$ at the end of period $N i+n$ with $0 \leq i \leq T-1$ and $1 \leq n \leq N$. Then the optimal claim policy for this policy holder is given by the following rule:

Claim for a damage if and only if $c(l, x)-f \geq D_{i}(\alpha, j, n, l, 1, x)$

Proof. Since the proof of this result is similar for the cases II, IV and VI we only give the proof for II.

A policy holder always decides what is most profitable for him and so he only claims if claiming provides him with less disutility than not claiming. This yields for case II using equation (4) that a policy holder will claim if and only if

$$
\exp (\alpha f) V_{i}(\alpha \beta, a(j), n+1, L(l, x), 1) \leq \exp (\alpha c(l, x)) V_{i}(\alpha \beta, j, n+1, L(l, x), 1) .
$$

By rewriting the above inequality and the definition of $D_{i}(\alpha, j, n, l, 1, x)$ the desired result now follows.

Observe, if a 'total loss' occurs, i.e. $x \geq \operatorname{tr}(l)$, that by (2) and (1)

$$
c(l, x)=w(l, x)=h(l)-C h(l) \frac{L-x}{L}=(1-C) h(l)+\frac{C x}{L} h(l),
$$

and so by Theorem 1 the policy holder claims if and only if

$$
x \geq \frac{L\left(D_{i}(\alpha, j, n, l, 1, x)+f-(1-C) h(l)\right)}{C h(l)} .
$$

Also for the cases I, III and IV, the policy holder has the opportunity to decide whether he will or will not be insured during the next period. Similarly as in the proof of Theorem 1 an optimal policy dictates the policy holder to decide the following:

Case I $(0 \leq i \leq T-1,1 \leq n \leq N-1, c=0)$. Applying equation (3) it follows almost immediately to effect an insurance if and only if

$$
\exp \left(\alpha \pi_{0}(j) \frac{N-n}{N}\right) V_{i}(\alpha \beta, j, n+1, L(l, x), 1) \leq V_{i}(\alpha \beta, j, n+1, L(l, x), 0) .
$$

Case III $(0 \leq i \leq T-2, n=N, c=0)$. Applying equation (5) it follows almost immediately to effect an insurance if and only if

$$
\exp \left(\alpha \pi_{0}(j)\right) V_{i+1}(\alpha \beta, b(j), 1, L(l, x), 1) \leq V_{i+1}(\alpha \beta, j, 1, L(l, x), 0) .
$$


Case IV $(0 \leq i \leq T-2, n=N, c=1)$. Applying equation (6) it follows almost immediately to effect an insurance in the case of having claimed for a damage $x$ if and only if

$$
\exp \left(\alpha \pi_{0}(a(j))\right) V_{t+1}(\alpha \beta, b(a(j)), 1, L(l, x), 1) \leq V_{i+1}(\alpha \beta, a(j), 1, L(l, x), 0) .
$$

Moreover, if it is optimal not to claim, the policy holder should effect the insurance if and only if

$$
\exp \left(\alpha \pi_{0}(j)\right) V_{i+1}(\alpha \beta, b(j), 1, L(l, x), 1) \leq V_{i+1}(\alpha \beta, j, 1, L(l, x), 0) .
$$

Observe the above inequalities also yield an upper bound on the premium a policy holder is willing to pay to effect or continue the vehicle damage insurance.

Up to now we have only given a description of the optimal claim behaviour of a policy holder. In the last part of this section we will derive a fast procedure to calculate this optimal policy. This will be illustrated by the calculations for case I. The calculations for the other cases can be performed similarly and are shown in Van Rijsoort (1990).

Observe first that for the last insurance year and $1<n \leq N, c=1, l<U$ and $j<J$, the policy holder claims every damage that is larger than or equal to his deduction $f$; as this is the last year he is insured he will not be troubled with a premium rise as a result of his claiming. So, we obtain for every $1 \leq n \leq N$ that

$$
D_{T-1}(\alpha, j, n, l, 1, x)=0 .
$$

In order to calculate the constants $D_{i}(\alpha, j, n, l, 1, x)$ given by (9) and (10) for $i \leq T-2$ we consider the cases shown in Table 4.

Before considering these cases we define the random variable $\underline{W}(l)$ as the 'total loss' cost resulting from an accident with a car that is $l$ subperiods old:

$$
0 \leq \underline{W}(l) \leq h(l) .
$$

In equation (1) we presented the realization $w(l, x)$ of random variable $\underline{W}(l)$. By this equation we obtain

$$
\underline{W}(l)=h(l)\left(1-C\left[\frac{L-\underline{X}}{L}\right]\right) .
$$

By the above definitions we can now write out the following expressions for case I.

Case 1. If this holds $(0 \leq i \leq T-1,1 \leq n \leq N-1, c=0)$, we obtain

$$
V_{i}(\alpha, j, n, l, 0)=\mathbb{E}\left(V_{i}\left(\alpha, j, n, l, 0, \underline{X}_{n}\right) 1_{\left\{\underline{X}_{n}<\operatorname{tr}(l)\right\}}\right)+\mathbb{E}\left(V_{i}\left(\alpha, j, n, l, 0, \underline{X}_{n}\right) 1_{\left\{\underline{X}_{n} \geq \operatorname{tr}(l)\right\}}\right) .
$$

Clearly by (3) and (2),

$$
\begin{aligned}
& \mathbb{E}\left(V_{i}\left(\alpha, j, n, l, 0, \underline{X}_{n}\right) 1_{\left\{\underline{X}_{n}<\operatorname{tr}(l)\right\}}\right) \\
& \quad=\mathbb{E}\left(\exp \left(\alpha \underline{X}_{n}\right) 1_{\left\{\underline{X}_{n}<\operatorname{tr}(l)\right\}}\right) * \min \left\{\begin{array}{l}
V_{i}\left(\alpha \beta, j, n+1, l^{*}, 0\right) \\
\exp \left(\alpha \pi_{0}(j)\left(\frac{N-n}{N}\right)\right) V_{i}\left(\alpha \beta, j, n+1, l^{*}, 1\right)
\end{array}\right.
\end{aligned}
$$

with $l^{*}=l(\bmod U)+1$. Applying again (2) and (3) it follows that

$$
\begin{aligned}
& \mathbb{E}\left(V_{i}\left(\alpha, j, n, l, 0, \underline{X}_{n}\right) 1_{\left\{\underline{X}_{n} \geq \operatorname{tr}(l)\right\}}\right) \\
& \quad=\mathbb{E}\left(\exp (\alpha \underline{W}(l)) 1_{\left\{\underline{X}_{n} \geq \operatorname{tr}(l)\right\}}\right) * \min \left\{\begin{array}{l}
V_{i}(\alpha \beta, j, n+1,1,0) \\
\exp \left(\alpha \pi_{0}(j)\left(\frac{N-n}{N}\right)\right) V_{i}(\alpha \beta, j, n+1,1,1)
\end{array}\right.
\end{aligned}
$$

and combining these two recurrent relations we obtain $V_{i}(\alpha, j, n, l, 0)$. Observe for a fast computation of $V_{i}(\alpha, j, n, l, 0)$ we need to have an analytical formula for the expectations. A way to achieve this is 
shown in Section 3. Similarly one can derive recurrent relations for the other cases shown in Table 4 but due to the same nature of these computations [cf. Van Rijsoort (1990)] they are omitted.

To conclude this section, we spend a few words on the way in which the values of $V_{i}(\alpha, j, n, l, c)$ and the critical claim sizes can be found. We will compute them iteratively, using the above equations, starting at time $N T$ and ending at time 1 . That is, for $1 \leq j \leq J, 1 \leq l \leq U$ and $c=0,1$ the values will be obtained in the following order:

$$
\begin{aligned}
& V_{T-1}(\alpha, j, N, l, c) \rightarrow D_{T-1}(\alpha, j, N-1, l, 1, x), x<\operatorname{tr}(l) \& x \geq \operatorname{tr}(l), \\
& V_{T-1}(\alpha, j, N-1, l, c) \rightarrow \ldots \rightarrow D_{0}(\alpha, j, 1, l, 1, x), x<\operatorname{tr}(l) \& x \geq \operatorname{tr}(l), \\
& V_{0}(\alpha, j, 1, l, c) .
\end{aligned}
$$

This algorithm computing the critical claim sizes uses $2(T-1) J N U$ steps and therefore operates in a time of $\mathrm{O}(T J N U)$.

In the next section we will analyse a vehicle damage insurance operative in the Netherlands.

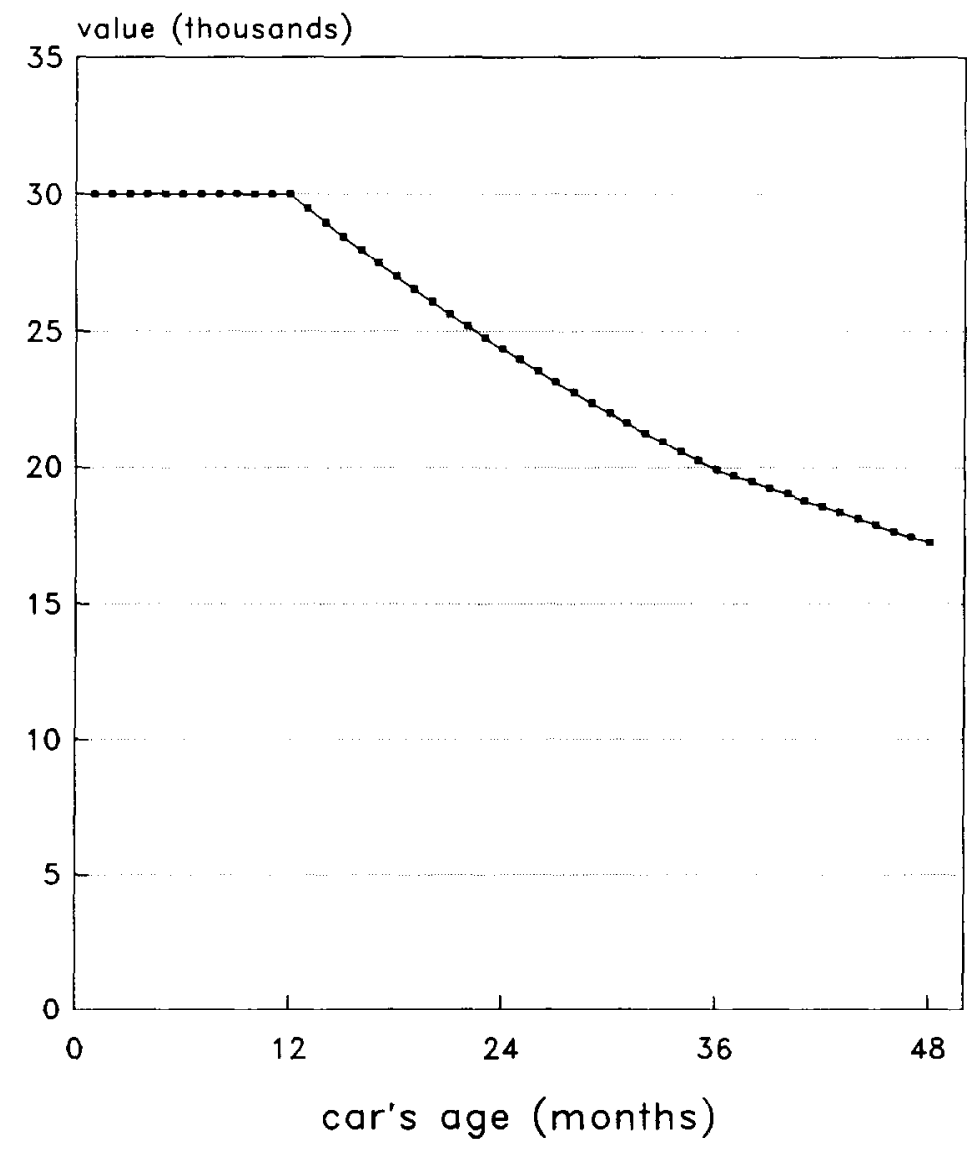

Fig. 1. Current values. 


\section{Results}

In this section we will present the results obtained by applying the model of Section 2 to the bonus-malus system given in Table 2 . Before showing the tables containing these results we will shortly discuss the distribution function and the parameters underlying the model.

We divide an insurance year in $N=12$ months and we assume that the probability $p_{n}$ of having an accident during period $n$ equals $\lambda / N$. For an automobile insurance a value of $\lambda=0.1$ accidents per year is reasonable.

Moreover, let the amount of damage $\underline{Y}_{n}$ have a truncated hyperexponential distribution [cf. Tijms (1986)]. As $0 \leq \underline{Y}_{n} \leq$ listprice, we truncate the distribution function at the listprice $L$.

The truncated hyperexponential distribution is a mixture of two truncated exponentials with different means. That is, $\underline{Y}_{n}$ is distributed with probability $q_{1}$ (respectively $1-q_{1}$ ) as a truncated exponential variable with parameter $\mu_{1}$ (respectively $\mu_{2}$ ) and truncation parameter $L$, i.e.

$$
F_{n}(x)=\mathbb{P}\left\{\underline{Y}_{n} \leq x\right\}=q_{1} \frac{1-\exp \left(-\mu_{1} x\right)}{1-\exp \left(-\mu_{1} L\right)}+\left(1-q_{1}\right) \frac{1-\exp \left(-\mu_{2} x\right)}{1-\exp \left(-\mu_{1} L\right)} .
$$

Also the normalization $q_{1} / \mu_{1}=\left(1-q_{1}\right) / \mu_{2}$ is used.

The reason for choosing this distribution is that it is easy to calculate the expectations derived in Section 2 and that it can fit the first two moments of a truncated distribution function with coefficient of variation bigger than 1 . The values of the chosen parameters are as follows:

$$
q_{1}:=0.723894318, \quad 1 / \mu_{1}:=1243.338025,1 / \mu_{2}:=3259.785616, \quad L:=30000,
$$

which corresponds to a mathematical expectation of Dfl.1800 and a squared coefficient of variation equal to 1.5 . We assume the parameters $q_{1}, \mu_{1}, \mu_{2}$ and $L$ to be constant throughout the entire duration of the decision process.

For reasons of simplicity we have taken the discount rate per period $\beta$ equal to 1 . The risk-aversion index $\alpha$ is chosen to be 0.0003 . With this $\alpha$, the policy holder will remain insured during the first two or three years that he owns his car. We assume that the policy holder keeps his car until a maximum age of $U=48$ periods $(=4$ years). The basic premium and the deduction respectively equal $\mathrm{Dfl} .1000$ and Dfl.300. The constant $C$ equals 0.5 .

Table 5

Optimal critical claim sizes (in Dfl.) for a horizon of 10 years, $l=24$ (for $n=1, \ldots, 12$ ), $i=0$ and no 'total loss'. (For comparison:

\begin{tabular}{|c|c|c|c|c|c|c|c|c|c|c|c|c|}
\hline$j, n$ & 1 & 2 & 3 & 4 & 5 & 6 & 7 & 8 & 9 & 10 & 11 & 12 \\
\hline 1 & 0 & 0 & 0 & 0 & 0 & 0 & 0 & 0 & 0 & 0 & 0 & 0 \\
\hline 2 & 0 & 0 & 0 & 0 & 0 & 0 & 0 & 0 & 0 & 0 & 0 & 0 \\
\hline 3 & 24 & 0 & 0 & 0 & 0 & 0 & 0 & 0 & 1 & 11 & 26 & 58 \\
\hline 4 & 317 & 298 & 283 & 270 & 257 & 243 & 234 & 261 & 291 & 320 & 348 & 374 \\
\hline 5 & 647 & 639 & 630 & 625 & 622 & 620 & 622 & 629 & 638 & 648 & 678 & 715 \\
\hline 6 & 957 & 954 & 951 & 953 & 962 & 972 & 985 & 997 & 1011 & 1026 & 1042 & 1061 \\
\hline 7 & 957 & 954 & 951 & 953 & 962 & 972 & 985 & 997 & 1011 & 1026 & 1042 & 1061 \\
\hline 8 & 1223 & 1230 & 1239 & 1249 & 1259 & 1269 & 1282 & 1298 & 1316 & 1336 & 1356 & 1378 \\
\hline 9 & 1201 & 1230 & 1239 & 1249 & 1259 & 1269 & 1282 & 1297 & 1314 & 1325 & 1330 & 1319 \\
\hline 10 & 1183 & 1209 & 1233 & 1256 & 1281 & 1306 & 1329 & 1317 & 1303 & 1293 & 1285 & 1280 \\
\hline 11 & 1083 & 1100 & 1118 & 1134 & 1149 & 1164 & 1175 & 1181 & 1187 & 1194 & 1182 & 1166 \\
\hline 12 & 955 & 966 & 978 & 986 & 989 & 991 & 991 & 992 & 992 & 992 & 992 & 990 \\
\hline 13 & 812 & 814 & 814 & 814 & 815 & 815 & 815 & 812 & 807 & 801 & 795 & 789 \\
\hline 14 & 904 & 907 & 909 & 909 & 910 & 911 & 911 & 909 & 905 & 900 & 894 & 890 \\
\hline 15 & 704 & 705 & 705 & 704 & 702 & 700 & 698 & 695 & 692 & 687 & 682 & 676 \\
\hline 16 & 742 & 743 & 743 & 743 & 741 & 740 & 738 & 735 & 732 & 727 & 722 & 717 \\
\hline 17 & 526 & 524 & 523 & 520 & 518 & 515 & 512 & 508 & 505 & 501 & 496 & 490 \\
\hline
\end{tabular}
$h(24):=$ Dfl.24349 and $\operatorname{tr}(24):=$ Dfl.20489.) 
Table 6

Optimal critical claim sizes (in Dfl.) for a horizon of 25 years, $l=24$ (for $n=1, \ldots, 12), i=0$ and no 'total loss'.

\begin{tabular}{|c|c|c|c|c|c|c|c|c|c|c|c|c|}
\hline$j, n$ & 1 & 2 & 3 & 4 & 5 & 6 & 7 & 8 & 9 & 10 & 11 & 12 \\
\hline 1 & 0 & 0 & 0 & 0 & 0 & 0 & 0 & 0 & 0 & 0 & 0 & 0 \\
\hline 2 & 850 & 856 & 862 & 867 & 873 & 878 & 884 & 890 & 896 & 901 & 907 & 912 \\
\hline 3 & 1568 & 1578 & 1588 & 1597 & 1606 & 1616 & 1625 & 1635 & 1644 & 1654 & 1663 & 1672 \\
\hline 4 & 2233 & 2246 & 2258 & 2271 & 2283 & 2296 & 2308 & 2321 & 2333 & 2346 & 2358 & 2369 \\
\hline 5 & 2833 & 2849 & 2863 & 2878 & 2893 & 2908 & 2922 & 2937 & 2952 & 2966 & 2981 & 2994 \\
\hline 6 & 3326 & 3343 & 3359 & 3375 & 3392 & 3408 & 3425 & 3441 & 3457 & 3474 & 3490 & 3504 \\
\hline 7 & 2508 & 2516 & 2524 & 2532 & 2540 & 2548 & 2555 & 2563 & 2571 & 2578 & 2586 & 2592 \\
\hline 8 & 2889 & 2898 & 2907 & 2917 & 2926 & 2935 & 2944 & 2953 & 2962 & 2971 & 2980 & 2988 \\
\hline 9 & 2199 & 2203 & 2206 & 2209 & 2211 & 2214 & 2217 & 2219 & 2222 & 2224 & 2226 & 2228 \\
\hline 10 & 1879 & 1878 & 1877 & 1875 & 1874 & 1872 & 1870 & 1869 & 1867 & 1865 & 1863 & 1860 \\
\hline 11 & 1557 & 1552 & 1547 & 1542 & 1537 & 1531 & 1526 & 1520 & 1514 & 1509 & 1503 & 1496 \\
\hline 12 & 1275 & 1267 & 1259 & 1251 & 1242 & 1234 & 1225 & 1216 & 1208 & 1199 & 1190 & 1180 \\
\hline 13 & 1005 & 997 & 989 & 981 & 972 & 954 & 956 & 947 & 938 & 930 & 921 & 912 \\
\hline 14 & 1078 & 1073 & 1068 & 1063 & 1058 & 1053 & 1047 & 1042 & 1037 & 1031 & 1026 & 1020 \\
\hline 15 & 815 & 810 & 805 & 800 & 794 & 789 & 784 & 778 & 773 & 767 & 762 & 756 \\
\hline 16 & 856 & 851 & 846 & 841 & 836 & 831 & 826 & 821 & 816 & 810 & 805 & 800 \\
\hline 17 & 595 & 590 & 585 & 580 & 575 & 570 & 564 & 559 & 554 & 549 & 544 & 539 \\
\hline
\end{tabular}

The function $h(l)$ denoting the car's value at age $l$ is defined by the following rules:

$l:=1, \ldots, 12: \quad$ no depreciation; $h(l):=$ listprice;

$l:=13, \ldots, 36: \quad 1,5 \%$ depreciation a month of the amount up to Dfl.15000,

$2 \%$ depreciation a month of the amount above Dfl.15000;

$l:=37, \ldots, 48$; linear depreciation until a residual value of Dfl.1000 at the age of 10 years [that is: $h(120)=$ Dfl.1000].

An illustration of the function $h(l)$ is given by Figure 1.

We now state the results.

In Tables 5 and 6 the optimal critical claim sizes $D$ are given in the case of no 'total loss' for horizons of 10 and 25 years. Since it is impossible to show them for all ages in one table the claim sizes are only given for a car of age 24 months in every period $n$ in the first year of the insurance, i.e. $i=0$. In the case of a 'total loss', the formula given by (12) will be negative. Therefore, in this case, the policy holder always claims.

If we consider Table 5 in combination with Table 8 , in which we describe whether or not a policy holder will be insured, we notice some remarkable observations. With the horizon of 10 years the car will no longer be insured if the policy holder finds himself in one of the bonus-malus class combinations 1 or 2. The critical claim sizes of these combinations (all equal to 0 ) are no longer important. In Table 5 we can also observe identical claim sizes for the combinations 6 and 7. Claiming damages while being in one of these combinations implies a transition to combination 1 or 2 and therefore leaving the insurance. Not claiming implies a transition to combination 9, regardless whether we are in combination 6 or in 7 . With a longer horizon, for instance 25 years like in Table 6 , these resemblances disappear, because now all policy holders will insure themselves for at least some years.

To give an idea of the course of the optimal critical claim sizes in relation to age $l$ Table 7 shows the mean critical claim sizes $\bar{D}$ for various values of $l$ and for a horizon of 10 years. We calculated these mean critical claim sizes in the following way: For example, consider Table 5. It shows the critical claim sizes for $l=24$. We obtain the mean critical claim size $\bar{D}$ for $l=24$ and $j=7$ by adding the claim sizes of the periods 1 to 12 of bonus-malus combination 7 and dividing this total amount by 12 .

For the bonus-malus combinations 5, 9, 13 and 17 Figure 2 gives an illustration of the connection between the optimal critical claim sizes and the age $l$ of the car at the first insurance period in the case 
of no 'total loss'. Figure 3 shows this optimal critical claim size in relation to the ultimate lower bound of a 'total loss' at age $l: \operatorname{tr}(l)$.

Looking at the results with regard to the optimal critical claim sizes we make the following remarks:

(1) When the horizon increases the optimal critical claim size also increases; due to the longer period during which he pays premium, it is important to the policy holder to be in a favourable bonus-malus class.

(2) In general, the bonus-malus combinations 3 to 11 have a higher critical claim size than the other bonus-malus combinations; Table 2 shows that claiming in these bonus-malus combinations gives rise to a relative high increase in the premium to be paid next year.

(3) The claim sizes increase with $n$ for $j=1, \ldots, 9$, and decrease with $n$ for $j=10, \ldots, 17$; in the beginning of a year, the probability of having more than one accident during that year is more alarming to the higher bonus-malus combinations than to the lower. The other way round, at the end of the year, not having claimed pays off better for the lower bonus-malus combinations (cf. Table 2).

(4) In the case of a 'total loss' the policy holder always claims.

(5) The critical claim sizes decrease with $l$ for $l=1, \ldots, 24$. This corresponds with the decrease of the car's current value. On the other hand, for the ages 25 to 48 the critical claim sizes show a rather strange course. This is imputed to the fact that the policy holder is no longer insured and pays for the damage, if any, himself. Therefore these claim sizes have no impact on the model.

Apart from the decision whether to claim or not the policy holder also has to choose between being and not being insured.

We distinguish between four starting-situations:

(a) The policy holder is insured and has a 'total loss';

(b) The policy holder is not insured and has a 'total loss';

(c) The policy holder is insured and has no 'total loss';

(d) The policy holder is not insured and has no 'total loss';

Table 7

Mean optimal critical claim sizes (in Dfl.) for a horizon of 10 years and no 'total loss'.

\begin{tabular}{|c|c|c|c|c|c|c|c|c|c|c|c|c|}
\hline \multirow[t]{2}{*}{$j$} & \multicolumn{12}{|l|}{$l$} \\
\hline & $l=4$ & 8 & 12 & 16 & 20 & 24 & 28 & 32 & 36 & 40 & 44 & 48 \\
\hline 1 & 0 & 0 & 0 & 0 & 0 & 0 & 0 & 0 & 0 & 0 & 0 & 0 \\
\hline 2 & 0 & 0 & 0 & 0 & 0 & 0 & 0 & 0 & 0 & 0 & 0 & 0 \\
\hline 3 & 86 & 49 & 20 & 9 & 9 & 10 & 16 & 20 & 58 & 117 & 152 & 143 \\
\hline 4 & 471 & 399 & 342 & 314 & 29.5 & 291 & 301 & 345 & 419 & 497 & 547 & 533 \\
\hline 5 & 872 & 795 & 732 & 684 & 654 & 643 & 655 & 709 & 798 & 902 & 956 & 941 \\
\hline 6 & 1244 & 1169 & 1100 & 1046 & 1010 & 989 & 995 & 1050 & 1152 & 1266 & 1324 & 1312 \\
\hline 7 & 1244 & 1169 & 1100 & 1046 & 1010 & 989 & 995 & 1050 & 1152 & 1266 & 1324 & 1312 \\
\hline 8 & 1551 & 1478 & 1410 & 1355 & 1315 & 1286 & 1287 & 1341 & 1448 & 1568 & 1627 & 1617 \\
\hline 9 & 1467 & 1429 & 1390 & 1346 & 1306 & 1276 & 1272 & 1322 & 1392 & 1455 & 1479 & 1477 \\
\hline 10 & 1362 & 1359 & 1347 & 1321 & 1299 & 1273 & 1261 & 1269 & 1303 & 1348 & 1360 & 1365 \\
\hline 11 & 1194 & 1195 & 1190 & 1183 & 1172 & 1153 & 1138 & 1136 & 1156 & 1176 & 1186 & 1192 \\
\hline 12 & 1003 & 1003 & 1002 & 1000 & 995 & 985 & 975 & 974 & 981 & 991 & 997 & 1001 \\
\hline 13 & 818 & 817 & 814 & 813 & 811 & 809 & 805 & 803 & 806 & 812 & 816 & 818 \\
\hline 14 & 914 & 914 & 912 & 910 & 908 & 905 & 900 & 898 & 900 & 904 & 909 & 913 \\
\hline 15 & 704 & 704 & 703 & 701 & 698 & 696 & 694 & 695 & 697 & 700 & 702 & 704 \\
\hline 16 & 743 & 744 & 742 & 740 & 738 & 735 & 733 & 734 & 736 & 739 & 741 & 743 \\
\hline 17 & 518 & 518 & 517 & 516 & 514 & 512 & 510 & 510 & 511 & 513 & 515 & 517 \\
\hline
\end{tabular}




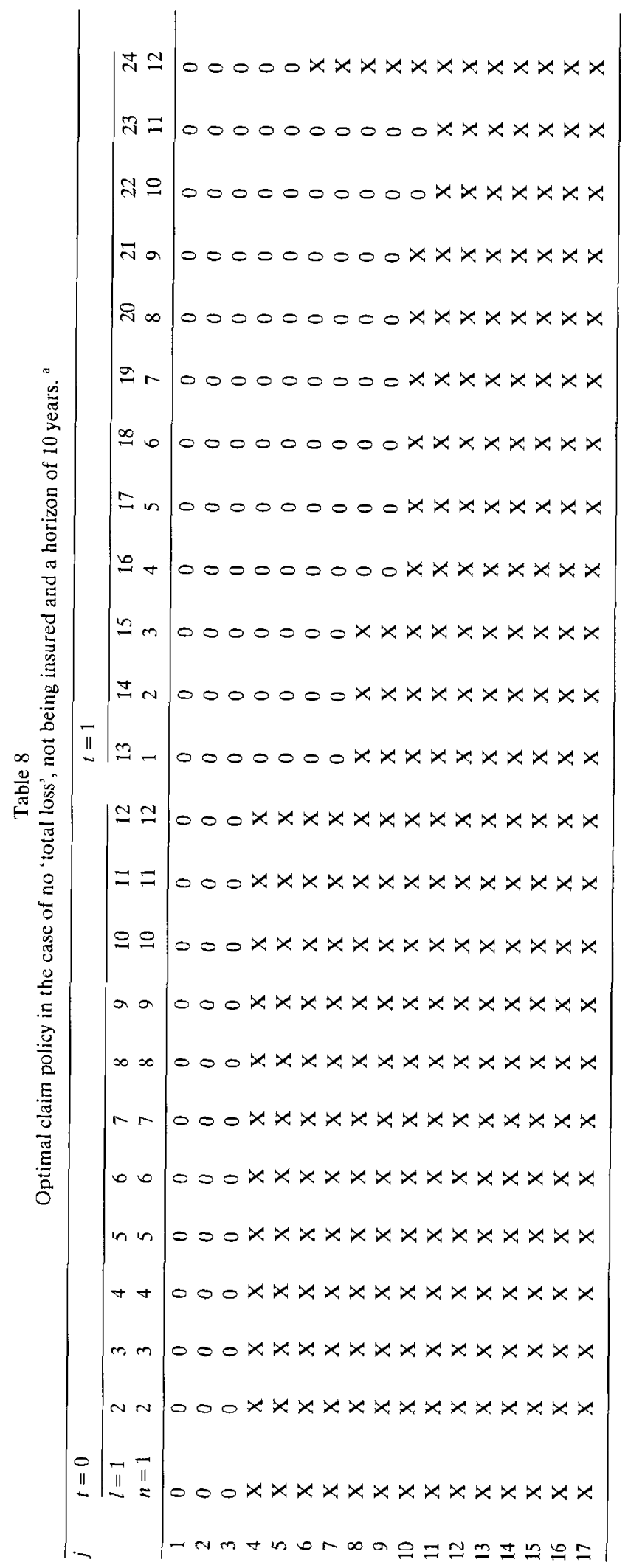




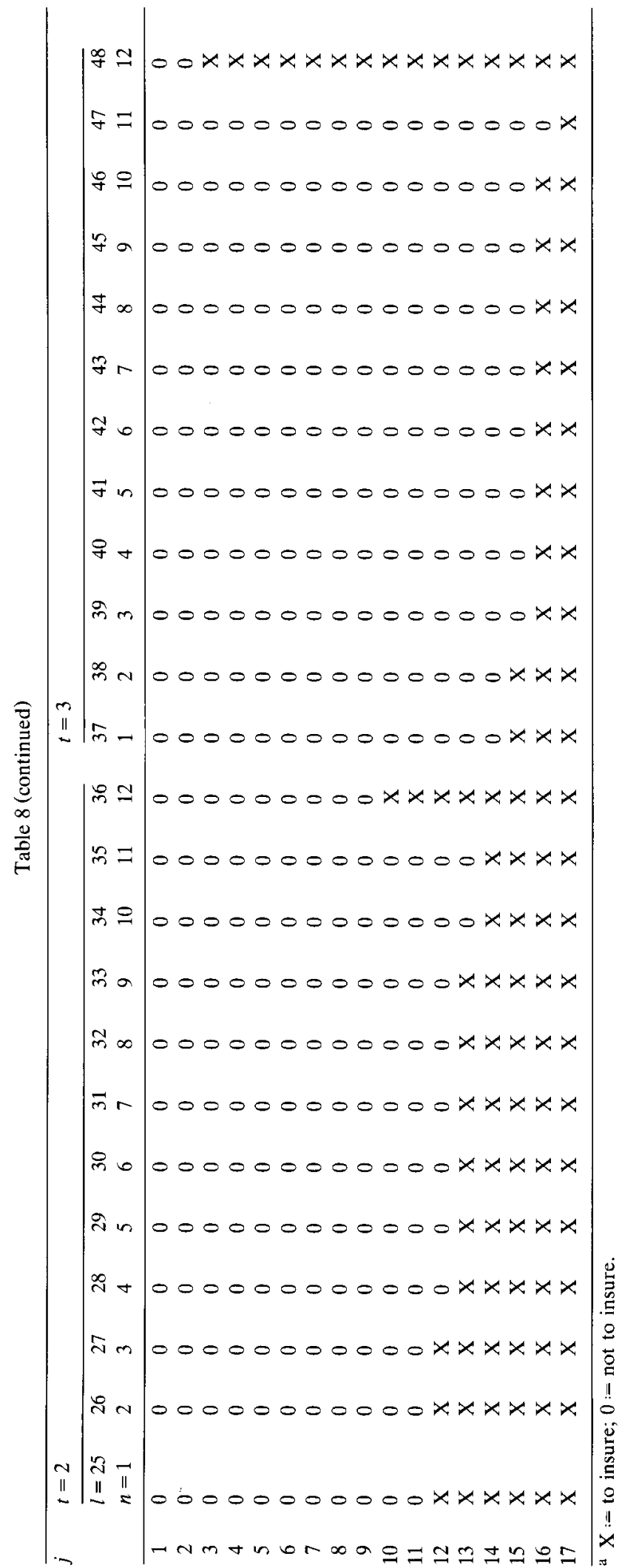



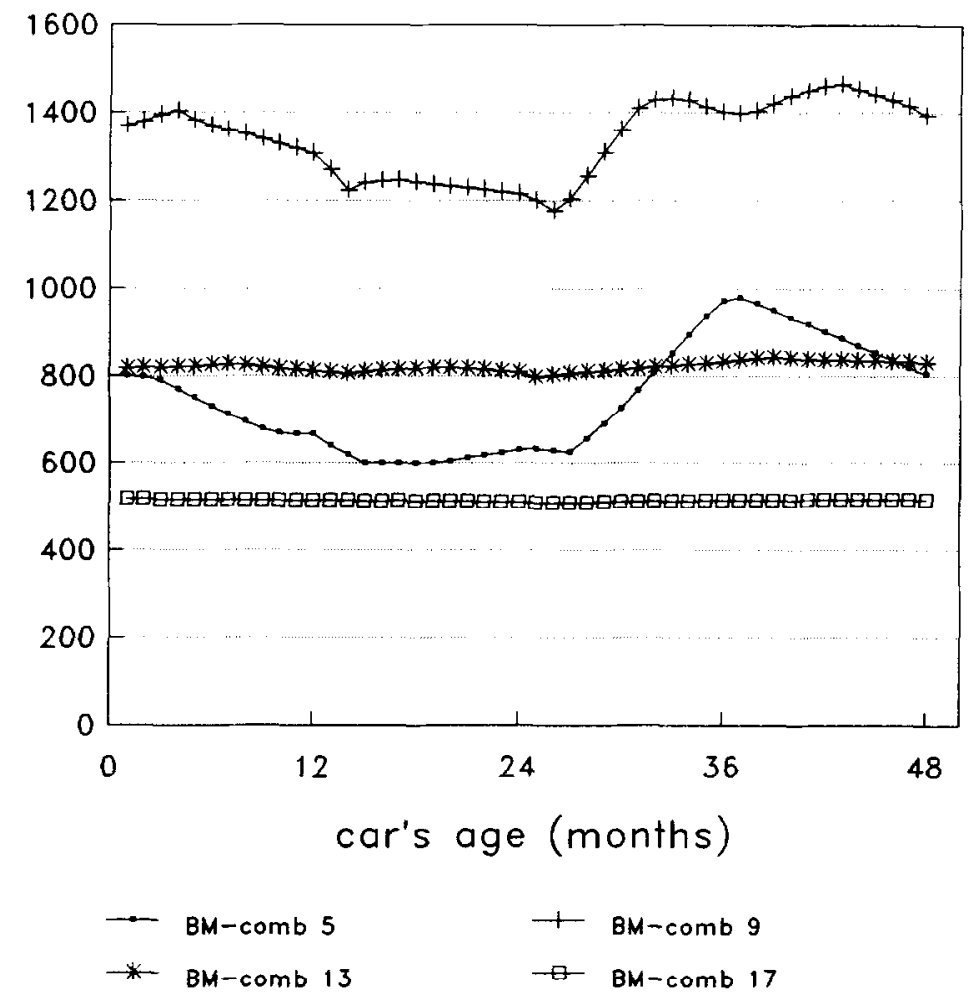

horizon 10 years

Fig. 2. Claim size $D$ for different BM-combinations at $t=0$ and $n=1$.

Table 9

Optimal claim policy in the case of no 'total loss', being insured and a horizon of 10 years.

\begin{tabular}{|c|c|c|c|c|c|c|c|c|}
\hline \multirow[t]{2}{*}{$j$} & \multicolumn{2}{|c|}{$t=0, n=12, l=12$} & \multicolumn{2}{|c|}{$t=1, n=12, l=24$} & \multicolumn{2}{|c|}{$t=2, n=12, l=36$} & \multicolumn{2}{|c|}{$t=3, n=12, l=48$} \\
\hline & $\begin{array}{l}\text { to } \\
\text { claim }\end{array}$ & $\begin{array}{l}\text { not to } \\
\text { claim }\end{array}$ & $\begin{array}{l}\text { to } \\
\text { claim }\end{array}$ & $\begin{array}{l}\text { not to } \\
\text { claim }\end{array}$ & $\begin{array}{l}\text { to } \\
\text { claim }\end{array}$ & $\begin{array}{l}\text { not to } \\
\text { claim }\end{array}$ & $\begin{array}{l}\text { to } \\
\text { claim }\end{array}$ & $\begin{array}{l}\text { not to } \\
\text { claim }\end{array}$ \\
\hline 1 & 0 & 0 & 0 & 0 & 0 & 0 & 0 & 0 \\
\hline 2 & 0 & 0 & 0 & 0 & 0 & 0 & 0 & 0 \\
\hline 3 & 0 & $X$ & 0 & 0 & 0 & 0 & 0 & $X$ \\
\hline 4 & 0 & $\mathrm{X}$ & 0 & 0 & 0 & 0 & 0 & $\mathrm{X}$ \\
\hline 5 & 0 & $\mathrm{X}$ & 0 & 0 & 0 & 0 & 0 & $X$ \\
\hline 6 & 0 & $\mathrm{X}$ & 0 & $\mathrm{X}$ & 0 & 0 & 0 & $\mathrm{X}$ \\
\hline 7 & 0 & $\mathrm{X}$ & 0 & $x$ & 0 & 0 & 0 & $\mathrm{X}$ \\
\hline 8 & 0 & $\mathrm{X}$ & 0 & $\mathrm{X}$ & 0 & 0 & 0 & $\mathrm{X}$ \\
\hline 9 & $\mathrm{x}$ & $\mathrm{X}$ & 0 & $\mathrm{X}$ & 0 & 0 & $X$ & $\mathrm{X}$ \\
\hline 10 & $\mathrm{x}$ & $\mathrm{x}$ & 0 & $\mathrm{X}$ & 0 & $\mathrm{x}$ & $\mathrm{X}$ & $x$ \\
\hline 11 & $X$ & $X$ & 0 & $X$ & 0 & $X$ & $X$ & $X$ \\
\hline 12 & $x$ & $X$ & $X$ & $\mathrm{X}$ & 0 & $X$ & $X$ & $X$ \\
\hline 13 & $x$ & $X$ & $X$ & $X$ & 0 & $X$ & $x$ & $X$ \\
\hline 14 & $\mathrm{X}$ & $X$ & $X$ & $X$ & 0 & $X$ & $X$ & $X$ \\
\hline 15 & $X$ & $X$ & $X$ & $X$ & $X$ & $X$ & $X$ & $X$ \\
\hline 16 & $x$ & $X$ & $X$ & $X$ & $X$ & $X$ & $X$ & $X$ \\
\hline 17 & $x$ & $X$ & $X$ & $\mathrm{X}$ & $X$ & $X$ & $X$ & $X$ \\
\hline
\end{tabular}




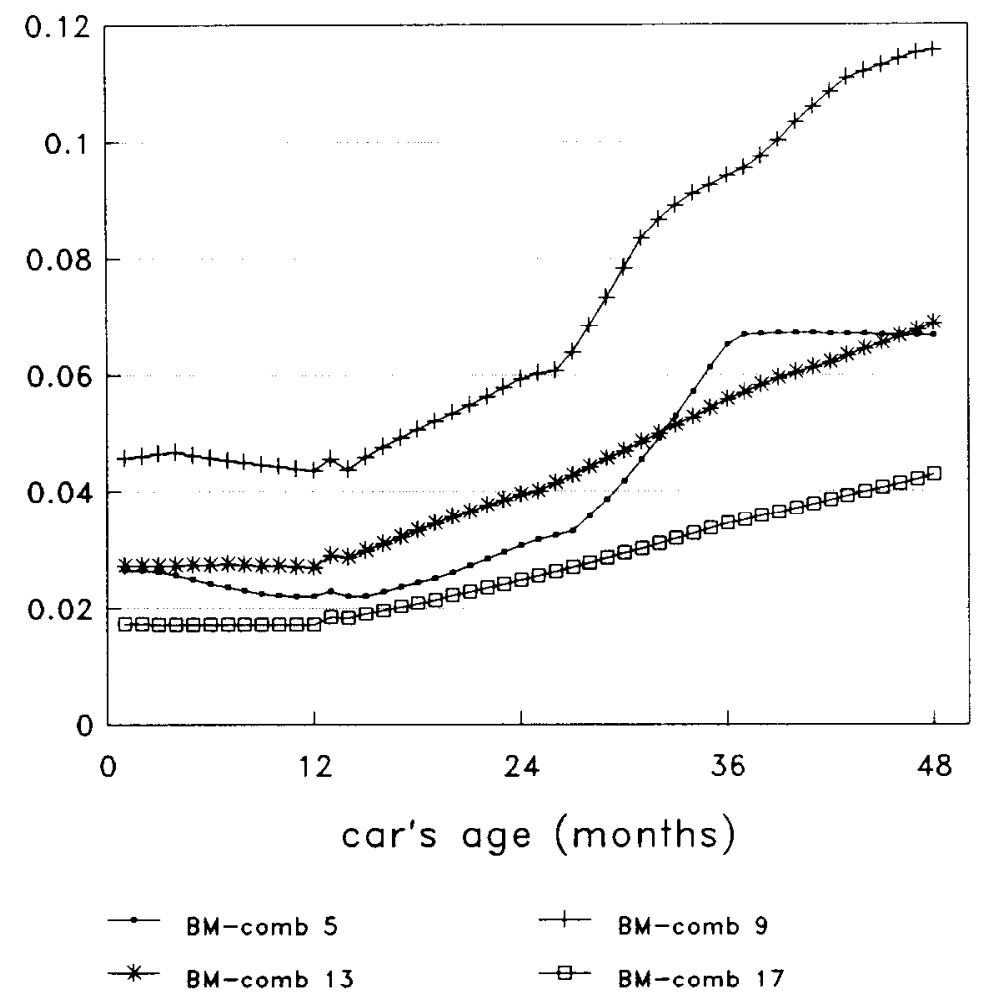

horizon 10 years

Fig. 3. Ratio $D / \operatorname{tr}(\mathrm{l})$ for different BM-combinations at $t=0$ and $n=1$.

In the situations (a) and (b) the choice whether to insure or not is easy. Since his car is a 'total loss', the policy holder buys a new one. Unless he is in one of the most unfavourable bonus-malus combinations (that is: $j=1,2$ or 3 ), the optimal insurance policy will always be to effect the insurance.

Assumed the policy holder does not have a 'total loss' [situations (c) and (d)] Tables 8 and 9 show the optimal insurance policy for the four years the policy holder is driving his car under the assumption that he buys his car at the first period and faces a horizon of 10 years. Remark that, if the policy holder is insured, he is only allowed to change his state of insurance at the end of the insurance year.

Table 8 shows the insurance policy in the case that he is at year $t$, period $n$, owns a car of age $l$, is in bonus-malus combination $j$ faces a no 'total loss' accident and is not insured; Table 9 shows the insurance policy in the case that he is insured.

With regard to the optimal insurance policy we make the following remarks:

(1) The older a car gets, the less it will be insured by the policy holder.

(2) In the case of a 'total loss' or when his car is 48 periods old the policy holder almost always effects an insurance. This is logical because he buys a new car the next period.

(3) The higher the bonus-malus combination, the longer a policy holder remains insured due to the lower cost of his premium.

(4) It is more favourable to insure at the end of a year and pay for the whole premium than to insure in the middle of a year and pay a partial premium.

To conclude we spend a few words on the sensitivity of the results in relation to the following five input parameters: $\alpha$, the basic premium, the deduction, the horizon, the listprice. 
When $\alpha$ increases, the policy holder is more afraid to take risks. Therefore, he remains insured up to a higher age $l$ and the optimal critical claim sizes increase.

The result of an increase of the basic premium is a policy holder who is less inclined to insure due to the higher cost of insurance. Moreover, the optimal critical claim sizes increase.

If the deduction increases, the optimal critical claim sizes decrease and the policy holder remains insured up to a lower age $l$. However, these changes are very small.

An increase of the horizon only gives rise to a change in results if it concerns a rather short period, for example from 10 to 25 years. In this case, the policy holder remains insured up to lower age $l$ and the optimal critical claim sizes increase; the policy holder is more careful because he looks upon a longer period of insurance. For horizons longer than about 25 years, the results stabilise.

Finally, when we increase the listprice, the policy holder insures up to a higher age $l$ (the basic premium did not change) and the optimal critical claim sizes increase (the cost of a damage, if any, is also increased).

\section{Acknowledgement}

The authors like to thank the anonymous referee for his constructive remarks which greatly improved the presentation of the paper.

\section{References}

Van Rijsoort, L. (1990). Optimal claim behaviour for insurances on hull and appurtenances. Master thesis, Econometric Institute, Erasmus University, Rotterdam.

Dellaert, N.P., J.B.G. Frenk, A. Kouwenhoven and B.S. van der Laan (1990). Optimal claim behaviour for third-party liability insurances or To claim or not to claim: that is the question. Insurance: Mathematics and Economics 9, 59-76.

Goudse Verzekeringen (1988). Tarief voor personenauto's.

Howard, R. and J. Matheson (1972). Risk-sensitive Markov decision processes. Management Science 18, 356-369.

Tijms, H.C. (1986). Stochastic Modelling and Analysis: A Computational Approach. Wiley, New York. 